\title{
Bayesian estimation of generalized half logistic type II doubly censored data
}

\author{
Reza Azimi \\ Department of Statistics, Parsabad Moghan Branch, Islamic Azad University, Parsabad Moghan, Iran \\ *Corresponding authorE-mail: azimireza1365@gmail.com,as.reza@hotmail.com
}

\begin{abstract}
This study considers the estimation problem for the parameter and reliability function of generalized half logistic distribution under Type II Doubly censored data. We use the Bayesian procedures (under asymmetric loss functions (Linex and general entropy) and symmetric loss function (squared-error)) to obtain the estimators of parameter and reliability function of generalized half logistic distribution .
\end{abstract}

Keywords: Generalized half logistic distribution, Bayesian estimation, Doubly censored, asymmetric loss function.

\section{Introduction}

Let us consider the generalized half logistic distribution whose cumulative distribution function, probability density function and reliability function are given, respectively, by

$$
\begin{aligned}
& F(x \mid \beta)=1-\left[\frac{2 e^{-x}}{1+e^{-x}}\right]^{\beta}, x>0, \beta>0 \\
& f(x \mid \beta)=\frac{\beta\left(2 e^{-x}\right)^{\beta}}{\left(1+e^{-x}\right)^{\beta+1}} \\
& R(t \mid \beta)=\left[\frac{2 e^{-t}}{1+e^{-t}}\right]^{\beta}
\end{aligned}
$$

For this model, Arora et al.[4] considered maximum likelihood estimators of the generalized half logistic distribution under type I progressive censoring with changing failure rates. Azimi et al. [2, 3] obtained Bayesian and E-Bayesian (expectation of the Bayesian estimate) estimators of the parameter and reliability function of generalized half logistic distribution by taking progressive type II censored sample using different loss functions such as LINEX, precautionary and entropy loss functions.

Consider a random sample of size $n$ from generalized half logistic distribution and let $x_{r}, \ldots, x_{s}$ be the ordered observations remaining when the $(r-1)$ smallest observations and the $(n-s)$ largest observations have been censored. The likelihood function of $\beta$ for type II doubly censored sample $x=\left(x_{r}, x_{r+1}, \ldots, x_{s}\right)$ is given by (see Fernandez [5] or kim and song [6] )

$$
L(\beta \mid \mathbf{X})=\frac{n !}{(r-1) !(n-s) !}[F(x)]^{r-1}[1-F(x)]^{n-s} \prod_{i=r}^{s} f\left(x_{i}\right) .
$$

\section{Likelihood, Prior and Posterior}

Substituting (1) and (2) into (4), the likelihood function becomes to be proportional to

$$
L(\beta \mid \mathbf{X}) \propto \beta^{s-r+1} e^{\beta\left(Q\left(u_{s}, u_{i}\right)\right)}\left[1-e^{\beta Q}\left(u_{r}\right)\right]^{r-1}
$$


Where,

$$
\begin{gathered}
U_{s}=\frac{2 e^{-x_{s}}}{1+e^{-x_{s}}}, U_{r}=\frac{2 e^{-x_{r}}}{1+e^{-x_{r}}}, U_{i}=\frac{2 e^{-x_{i}}}{1+e^{-x_{i}}} \\
Q\left(u_{s}, u_{i}\right)=(n-s) \ln U_{s}+\sum_{i=r}^{s} \ln U_{i}, \quad \dot{Q}\left(u_{r}\right)=\ln U_{r}
\end{gathered}
$$

It is assumed that the parameter $\beta$ has a gamma prior distribution with the shape and scale parameters as $a$ and $b$, respectively and it has the PDF

$$
\pi(\beta)=\frac{b^{a}}{\Gamma(a)} \beta^{a-1} e^{-b \beta}
$$

Combining (5) and (6) the posterior density function of $\beta$ takes the form

$$
\pi(\beta \mid x)=\frac{\left(b-Q\left(u_{s}, u_{i}\right)\right)^{a^{*}}}{\Gamma\left(a^{*}\right) G_{\dot{Q}}\left(Q\left(u_{s}, u_{i}\right), a^{*}\right)} \beta^{a^{*}-1} e^{-\beta\left(b-Q\left(u_{s}, u_{i}\right)\right)}\left[1-e^{\beta \dot{Q}\left(u_{r}\right)}\right]^{r-1}
$$

where,

and

$$
a^{*}=s-r+a+1
$$

$$
G_{\dot{Q}}\left(Q\left(u_{s}, u_{i}\right), a^{*}\right)=\left(b-Q\left(u_{s}, u_{i}\right)\right)^{a^{*}} \sum_{k=0}^{r-1} \frac{(-1)^{k}\left(\begin{array}{c}
r-1 \\
k
\end{array}\right)}{\left(b-Q\left(u_{s}, u_{i}\right)-k \dot{Q}\left(u_{r}\right)\right)^{a^{*}}}, \quad G_{0}=1 .
$$

Substituting $\beta=\frac{-\ln R}{\ln U_{t}}$ into (7), we can obtain the posterior density function of $R=R(t)$ as

$$
\pi(R \mid \mathbf{X})=\left(\frac{b-Q\left(u_{s}, u_{i}\right)}{\ln U_{t}}\right)^{a^{*}} \frac{R^{\frac{b-Q\left(u_{s}, u_{i}\right)}{\ln U_{t}}}-1}{\Gamma\left(a^{*}\right) G_{\dot{Q}}\left(Q\left(u_{s}, u_{i}\right), a^{*}\right)}(-\ln R)^{a^{*}-1}\left[1-R^{-\frac{Q\left(u_{r}\right)}{\ln U_{t}}}\right]^{r-1}
$$

where $0<R<1$ and $U_{t}=\frac{1+e^{-t}}{2 e^{-t}}$.

\section{Bayesian estimation with squared-error loss function}

Under squared-error loss, the Bayes estimator of $\beta$ is the mean of the posterior density given by

$$
\hat{\beta}_{s e}=\frac{a^{*}}{b-Q\left(u_{s}, u_{i}\right)} \frac{G_{\dot{Q}}\left(Q\left(u_{s}, u_{i}\right), a^{*}+1\right)}{G_{\dot{Q}}\left(Q\left(u_{s}, u_{i}\right), a^{*}\right)} .
$$

Under squared-error loss function, the Bayesian estimators of $R(t)$ is given by

$$
\hat{R}_{s e}=\left(\frac{b-Q\left(u_{s}, u_{i}\right)}{b-Q\left(u_{s}, u_{i}\right)+\ln U_{t}}\right)^{a^{*}} \frac{G_{\dot{Q}}\left(Q\left(u_{s}, u_{i}\right)+\ln U_{t}, a^{*}\right)}{G_{\dot{Q}}\left(Q\left(u_{s}, u_{i}\right), a^{*}\right)} .
$$

\section{Bayesian estimation with LINEX loss function}

The LINEX loss function for $\beta$ can be expressed as the following proportional

$$
L(\Delta) \propto \exp (c \Delta)-c \Delta-1 ; \quad c \neq 0
$$

where $\Delta=(\hat{\beta}-\beta)$ and $\hat{\beta}$ is an estimate of $\beta$. The Bayes estimator of $\beta$, denoted by $\hat{\beta_{L}}$ under the LINEX loss function is given by

$$
\hat{\beta_{l i}}=-\frac{1}{c} \ln E_{\beta}[\exp (-c \beta)]
$$

Under LINEX loss function, we obtain Bayesian estimator of the parameter $\beta$, and reliability function $R(t)$, as the following forms

$$
\begin{gathered}
\hat{\beta}_{l i}=-\frac{1}{c} \ln \left[\left(\frac{b-Q\left(u_{s}, u_{i}\right)}{b-Q\left(u_{s}, u_{i}\right)+c}\right)^{a^{*}} \frac{G_{\dot{Q}}\left(Q\left(u_{s}, u_{i}\right)+c, a^{*}\right)}{G_{\dot{Q}}\left(Q\left(u_{s}, u_{i}\right), a^{*}\right)}\right] \\
\hat{R}_{l i}=-\frac{1}{c} \ln \left[\sum_{j=0}^{\infty} \frac{(-1)^{j} c^{j}}{j !}\left(\frac{b-Q\left(u_{s}, u_{i}\right)}{b-Q\left(u_{s}, u_{i}\right)+j \ln U_{t}}\right)^{a^{*}} \frac{G_{\dot{Q}}\left(Q\left(u_{s}, u_{i}\right)+j \ln U_{t}, a^{*}\right)}{G_{\dot{Q}}\left(Q\left(u_{s}, u_{i}\right), a^{*}\right)}\right]
\end{gathered}
$$




\subsection{Bayesian estimation with general entropy loss function}

In many practical situations, it appears to be more realistic to express the loss in terms of the ratio $\frac{d}{\beta}$. In this case, Calabria and Pulcini [1] point out that a useful asymmetric loss function is the general entropy loss function,

$$
L(\delta)=\left[\delta^{q}-q \ln (\delta)-1\right], \delta=\frac{d}{\beta} .
$$

The magnitude of $q$ reflects the degree of asymmetry. The Bayes estimator of $\beta$ under the GELF is given by

$$
\hat{\beta}_{g e}=\left[E\left(\beta^{-q} \mid \mathbf{x}\right)\right]^{-\frac{1}{q}}
$$

Therefore the Bayesian estimators of parameter $\beta$ and and reliability function $R(t)$ under general entropy loss function are given by

$$
\hat{\beta}_{g e}=\left[\frac{\Gamma\left(a^{*}-q\right)}{\Gamma\left(a^{*}\right)} \frac{G_{\dot{Q}}\left(Q\left(u_{s}, u_{i}\right), a^{*}-q\right)}{G_{\dot{Q}}\left(Q\left(u_{s}, u_{i}\right), a^{*}\right)}\right]^{-\frac{1}{q}} \frac{1}{b-Q\left(u_{s}, u_{i}\right)}
$$

and

$$
\hat{R}_{g e}=\left(\frac{b-Q\left(u_{s}, u_{i}\right)}{b-Q\left(u_{s}, u_{i}\right)-q \ln U_{t}}\right)^{-\frac{a^{*}}{q}}\left(\frac{G_{\dot{Q}}\left(Q\left(u_{s}, u_{i}\right)-q \ln U_{t}, a^{*}\right)}{G_{\dot{Q}}\left(Q\left(u_{s}, u_{i}\right), a^{*}\right)}\right)^{-\frac{1}{q}} .
$$

\section{$5 \quad$ Numerical example}

The following type II doubly censored sample of size $n=12(r=3, s=11)$ is generated from generalized half logistic distribution with $\beta=2$.

$$
\begin{gathered}
-,-, 0.1653687,0.1747466,0.4653450,0.6980732,0.7917320, \\
0.8060390,0.9330400,1.2799765,1.6043055,-
\end{gathered}
$$

The first two failure times and the last one are censored. We used the above sample to compute the Bayesian estimators of parameter $\beta$ and reliability function $R(t)$. In all above cases the prior parameters chosen as $a=2$ and $b=1$, which yield the generated value of $\beta=2$ as the true value. The true values of $R(t)$ in $t=0.5$ is obtained $R(0.5)=0.5701478$ The results obtained are given in Table 1 .

Table 1: Bayesian Estimators of parameter $\beta$ and reliability function $R(t)$.

\begin{tabular}{ccc|ccc}
\hline$\hat{\beta}_{s e}$ & $\hat{\beta}_{l i}(c=0.1)$ & $\hat{\beta}_{g e}(q=1)$ & $\hat{R}_{s e}$ & $\hat{R}_{l i}(c=0.1)$ & $\hat{R}_{g e}(q=1)$ \\
\hline 2.007478 & 1.992129 & 1.853002 & 0.5757593 & 0.5750276 & 0.5618253 \\
\hline
\end{tabular}

\section{Conclusions}

In this paper, we have presented the Bayesian estimates (with different loss funccions) of the parameter $\beta$, and reliability functions of generalized half logistic distribution based on type II doubly censored samples. A numerical example in section 5 to illustrate our approach.

\section{References}

[1] R. Calabria, G. Pulcini, Point estimation under asymmetric loss functions for left-truncated exponential samples, Communications in Statistics Theory and Methods 25(1996) 585-600.

[2] R. Azimi, F. Yaghmaei, M. Babanezhad, Bayesian Estimation of Generalized Half Logistic Distribution Under Progressive Type-II Censored Data. Applied Mathematical Sciences 6(2012) 106, 5253-5261.

[3] R. Azimi, F. Yaghmaei, B. Fasihi, E-Bayesian estimation based on generalized half Logistic progressive type-II censored data. International Journal of Advanced Mathematical Sciences, 1 (2) (2013) 56-63. 
[4] S.H. Arora, G.C. Bihmani, M.N. Patel, Some Results on Maximum Likelihood Estimators of Parameters of Generalized Half Logistic Distribution under Type-I Progressive Censoring with Changing Failure Rate. International Journal Of Contemporary Mathematical Sciences 5(2010) (14), 685-698.

[5] Fernandez, A. J.(2000). Bayesian inference from type II censored Rayleigh data. Statistics and Probability Letters, 48, 393-399.

[6] C. kim and S. song, Bayesian estimation of the parameters of the generalized exponential distribution from doubly censored samples, Stat Papers DOI 10.1007/s00362-008-0142-3. 